\title{
Harnwegserkrankungen: Hoffnungsträger Botulinumtoxin
}

— Bislang kann Botulinumtoxin zur Behandlung von Erkrankungen wie überaktive Blase $(\mathrm{OAB})$, Harninkontinenz oder interstitielle Zystitis nur „off-label“ eingesetzt werden. Unbeirrt davon forschen Wissenschaftler an den verschiedenen Anwendungsmöglichkeiten.

Dr. Douglas Tincello, Leicester, präsentierte die Ergebnisse der bislang größten Studie zum Einsatz von Botulinumtoxin A bei idiopathischer Detrusorhyperaktivität (definiert als mindestens acht Miktionen und zwei Drangepisoden binnen 24 Stunden). 240 Frauen wurden randomisiert. Nach sechs Monaten waren $30 \%$ der Frauen, die 200 U Botulinumtoxin A erhielten, kontinent, im Vergleich zu $11 \%$ in der Placebogruppe. Zudem verringerten sich die Miktionshäufigkeit von 12,0 auf 8,7 (Placebo: 11,3 auf 10,5), die Anzahl der Drangepisoden von 8,1 auf 4,4 (Placebo: 8,2 auf 6,8) sowie die Anzahl der Inkontinenzepisoden von 6,5 auf 3,1 (Placebo 6,3 auf 5,7). Bei 16\% der Frauen aus der Botulinumtoxin-Gruppe war eine intermittierende Selbstkatheterisierung notwendig, im Gegensatz zu $4 \%$ in der Placebo-Gruppe.

Ähnlich erfolgreich verlief eine Studie zum Einsatz von Botulinumtoxin A bei Harninkontinenz, die Dr. Francisco Cruz, Porto, vorstellte. 275 Patienten, die neben multipler Sklerose oder Rückenmarksverletzungen an neuropathischer Detrusorhyperaktivität litten, wurden randomisiert und erhielten entweder $200 \mathrm{U}$ Botulinumtoxin A, $300 \mathrm{U}$ Botulinumtoxin A oder Placebo. Nach sechs Wochen nahm die Anzahl der Inkontinenzepisoden von 33,5 auf 11,7 (200 U), 14,1 (300 U) und 20,3 (Placebo) ab. Auch in weiteren Parametern schnitten die beiden BotulinumtoxinA-Dosierungen signifikant besser ab als Placebo, zwischen den beiden Dosie- rungen gab es keine relevanten Unterschiede. Cruz empfahl daher, in Zukunft eine maximale Dosis von $200 \mathrm{U}$ zu verwenden. Diese sei genauso wirksam, aber kostengünstiger und nebenwirkungsärmer. Schon in früheren Studien lagen „ideale“ Dosen meist zwischen $100 \mathrm{U}$ und 200 U, wie Prof. Christopher Chapple, Sheffield, berichtete.

Auch in der Behandlung des chronischen Blasenschmerzsyndroms (interstitielle Zystitis, BPS/IC) mit Botulinumtoxin gibt es Fortschritte zu verzeichnen. Prof. Antonella Giannantoni, Perugia, präsentierte einen Überblick über mehrere kleine Studien, bei denen sich die BPS/IC-Symptome der Patienten über einen Zeitraum von mehreren Monaten subjektiv besserten. In zukünftigen Studien soll nun herausgefunden werden, wann der beste Zeitpunkt für eine erneute Injektion ist.

Eine weitere Indikation für Botulinumtoxin könnte die Behandlung von Symptomen des unteren Harntrakts (LUTS) im Rahmen einer benignen Prostatahyperplasie (BPH) sein. In einer kleinen Pilotstudie wurde 10 Patienten mit LUTS 200-300 U Botulinumtoxin A in die Transitionalzone der Prostata injiziert. Nach drei Monaten sanken bei diesen Patienten Prostatavolumen, IPP-Score und Restharnvolumen, maximale Harnflussrate und Lebensqualität nahmen zu, jeweils signifikant im Vergleich zur Placebogruppe. Nach Ansicht der Autoren könnte die Botulinumtoxin-Injektion eine Option für BPH-Patienten sein, die auf eine Behandlung mit Alphablockern und 5-Alpha-Reduktase-Hemmern nicht ansprechen oder die eine Operation scheuen.

slx

P 325, P 439, P 579, P 581, SP 3

\section{WHO-Checkliste bei Operationen befolgen}

— Im Operationssaal sind die Teams während des Eingriffs oft mehreren Störquellen ausgesetzt. In einer in Wien vorgestellten Studie wurde untersucht, wie häufig Unterbrechungen tatsächlich sind und welchen Einfluss das auf die Sicherheit des Eingriffs hat.

Ein Chirurg und ein Psychologe beobachteten insgesamt 20 Operationen von eingespielten Teams. Die Unterbrechungen wurden vom Psychologen mit einem validierten Verfahren in drei Gruppen eingestuft (kommunikationsbedingte, ausrüstungsbedingte oder andere Ursache) und je nach Ausmaß auf einer Skala von 1-9 bewertet. Der Chirurg protokollierte Fehler, die das Patientenwohl potenziell gefährden, mittels einer validierten Checkliste aus dem „observational teamwork assessment for surgery“ (OTAS).

Die Eingriffe dauerten im Mittel insgesamt 68 Minuten, die reine Operationszeit betrug 29 Minuten. Durchschnittlich wurden die Operateure sechs mal pro Stunde unterbrochen (137 Fälle, davon 85 kommunikationsbedingt). Am häufigsten wurden die Prozeduren durch Kommentare von OP-Schwestern unterbrochen, am gravierendsten waren Störungen durch Externe und den Operateur selbst. Häufige Unterbrechungen korrelierten signifikant mit einem schlechten Abschneiden beim OTAS-Test.

Studienautor Dr. Shabnam Undre empfiehlt, sich bei Operationen an die WHOCheckliste (www.who.int/patientsafety/ safesurgery) zu halten, um das Patientenwohl nicht unnötig zu gefährden. In weiteren Studien soll herausgefunden werden, ob sich mit der WHO-Checkliste kommunikationsbedingte Unterbrechungen reduzieren lassen.

slx

P 110 